
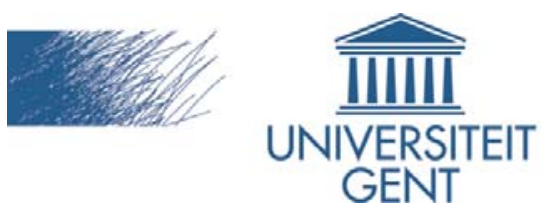

biblio.ugent.be

The UGent Institutional Repository is the electronic archiving and dissemination platform for all UGent research publications. Ghent University has implemented a mandate stipulating that all academic publications of UGent researchers should be deposited and archived in this repository. Except for items where current copyright restrictions apply, these papers are available in Open Access.

This item is the archived peer-reviewed author-version of:

Assistive Smartphone for People with Special Needs: the Personal Social Assistant

Steven Verstockt, Sofie Van Hoecke, Onciny Meyfroodt, Rik Van de Walle

Abstract Booklet of Medicine 2.0, p. 69, 2010.

To refer to or to cite this work, please use the citation to the published version:

Steven Verstockt, Sofie Van Hoecke, Onciny Meyfroodt, Rik Van de Walle (2010), Assistive Smartphone for People with Special Needs: the Personal Social Assistant, Abstract Booklet of Medicine 2.0, p. 69 


\title{
Assistive Smartphone for People with Special Needs: the Personal Social Assistant
}

\author{
Steven Verstockt, Electronics and Information Technology Lab, University College West Flanders, \\ Ghent University Association, Kortrijk, Belgium \\ Sofie Van Hoecke, Electronics and Information Technology Lab, University College West Flanders, \\ Ghent University Association, Kortrijk, Belgium \\ Onciny Meyfroodt, Electronics and Information Technology Lab, University College West Flanders, \\ Ghent University Association, Kortrijk, Belgium \\ Rik Van de Walle, ELIS- Multimedia Lab, Ghent University - IBBT, Gent, Belgium
}

\section{Background}

The growing usage of ICT in everyday life, by means of devices such as mobile phones, smart phones and GPS, has changed society. In a world getting more mobile every day, being able to use these devices is the key to integration. This is no easy matter for people with certain disabilities and special needs. For many of them, access to the benefits of mobile technology is limited because this technology is mostly designed for the younger generation, who can easily handle complicated devices. Elderly and disabled people encounter difficulties in using those devices: they cannot operate the controls, cannot obtain information from the device or do not understand how to operate it.

\section{Objective}

The Personal Social Assistant (PSA) described in this paper is an adaptation tool that shows how elderly people and cognitive, visual, and motor impaired persons can also make use of contemporary mobile devices if the interface is brought to their level. The PSA makes existing devices more accessible without modifications to internal hardware or software. The PSA is based on a new user interface strategy that makes it easier for people to interact with smart phones and other types of mobile technology. It is a great example of how ICT can be used by people who, at this moment, cannot use it at all. Everyone should have access to ICT and the major goal of the PSA is to bring it right to them.

\section{Methods}

One of the primary goals is to support PSA on existing smart phones. Therefore, we followed the "Design for All" design principle in order to support universal access. This way, we widen access to mobile devices to groups which might otherwise be unable to enjoy these benefits when dedicated, expensive assistive devices should be used.

\section{Results}

We developed a user-centric interface that is accessible and adaptable for everyone. This interface uses services in the background that are already available on the device, extended with specific addons, i.e. extensions that developed to make existing applications more intuitive to use. The technological innovations making this all possible are scalable interfaces, SMS/MMS push technology, full text-to-speech, and icon and photo-based navigation. As this interface sits on top of devices that are commercially available, the production costs stay low and the time-to-market becomes very short.

\section{Conclusion}

The overwhelming majority of mobile devices are being developed primarily for able-bodied young users. The Personal Social Assistant is designed so that people with certain disabilities can also use these mobile devices very easy. Meaningful icon menus, text-to-speech, the scalable interface and the easy-to-use touch screen give the device a great usability and accessibility. Because everyday technology is used, it is easy for support people to give support and advice on how to use the device. But perhaps the most important advantage of using commercially available devices is that disabled people will not be judged as different when using them. Using the same smart phone like everybody else provides them with a strong feeling of self worth. 